\title{
Korean Complementary Acupuncture Treatment Associated Severe Facial Infection Case
}

\author{
Kwan Sub Kim¹, Wu Seop Lee ${ }^{2}$, Sung Min Shim³ , Su Hyun Kwak4, Yong Min Kim5, So Young Ji² \\ Departments of ${ }^{1}$ Anesthesiology and Pain Medicine and ${ }^{2}$ Plastic and Reconstructive Surgery, Dongkang Medical Center, Ulsan; ${ }^{3}$ Department of Anesthesiology \\ and Pain Medicine, Gangneung Asan Medical Center, University of Ulsan College of Medicine, Gangneung; ${ }^{4}$ Department of Rehabilitation Medicine, Semin \\ Hospital, Ulsan; ${ }^{5}$ The Hong Kong University of Science and Technology, Hong Kong
}

\begin{abstract}
Acupuncture, an Asian traditional medicine, has been studied and applied in Northeastern Asia including Korea for thousands of years. This traditional medicine is believed to have significant improvements in reducing pain and swelling, but there have been reported cases of various side effects, especially infections. Infected wounds by an acupuncture treatment have caused sepsis in some cases. In this report, we present the case of successful treatment of infected wounds caused by the Korean traditional acupuncture as the result of a collaboration between plastic surgery and anesthetics departments.
\end{abstract}

Keywords: Acupuncture; Abscess; Drainage

\section{Introduction}

Head and neck are less likely to be affected by chronic infections due to their abounding blood flow. Although some odontogenic, sinusitis-induced and iatrogenic infection cases are reported, the facial abscess occurrences are known to be very low [1].

Traditional acupuncture treatments, commonly adopted throughout the Northeast-Asian region, are empirically believed to have an effect in pain and edema control. They are thus widely applied to deal with diverse indications including convulsion and neuropathy [2]. However, side effects of infection, tetanus, allergy, and fainting have been reported, thus caution needs to be taken at the time of treatment to avoid such symptoms.

In this case, the severe abscess was developed in a healthy patient (without any history of diabetes mellitus and immunocompromised diseases) after a single acupuncture treatment that was initiated after a sporadic eyelid tremor at a local traditional clinic. At our hospital (Dongkang Medical Center), the patient was able to fully recover after the surgical drainage with a month of conservative treatment including antibiotics.

This report will illustrate the case of an infection, which the immediate treatment is necessary to improve the patient's condition without much adverse effect.

\section{Case}

A 65-year-old female patient was admitted to the hospital due to almost 10 days of swelling, redness, and tenderness on her left periocular and temporal area. Before the admission, the patient had a Korean acupuncturist treatment on her sporadic eyelid

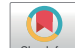

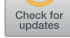 \\ Case Report \\ Received: December 10, 2018 \\ Revised: February 20, 2019 \\ Accepted: February 20, 2019 \\ Corresponding author: \\ So Young Ji, M.D. \\ Department of Plastic and Reconstructive \\ Surgery, Dongkang Medical Center, \\ 239 Taehwa-ro, Jung-gu, Ulsan 44455, Korea \\ Tel: $+82-52-241-1325$ \\ Fax: +82-52-241-1180 \\ E-mail: bsbaiksy@naver.com
}

This is an Open Access article distributed under the terms of the Creative Commons Attribution Non-Commercial License (http://creativecommons.org/licenses/by-nc/4.0/) which permits unrestricted non-commercial use, distribution, and reproduction in any medium, provided the original work is properly cited.

(c) 2019 Korean Wound Management Society 
tremor. After the first acupuncture treatment on her left periorbital area, the patient started to suffer from fever and persisting swelling and tenderness on her left cheek. Eight days after the acupuncture procedure, she was admitted to a local hospital. Her initial laboratory examination revealed a C-reactive protein (CRP) level of $4.94 \mathrm{mg} / \mathrm{dL}$ and the white blood cell (WBC) level at $12,500 / \mu \mathrm{L}$, and body temperature of $38.2^{\circ} \mathrm{C}$. The patient was diagnosed with systemic inflammatory response syndrome (SIRS) based on the results of the blood sample and the body temperature. The patient was hospitalized for 3 days at the local hospital, but the persisting symptoms made the patient transfer to our hospital with a local hospital's note. We have admitted the patient after 12 days since the first acupuncture treatment.

At first sight, severe tenderness, swelling, and redness were observed at the outpatient clinic at our hospital (Fig. 1). A $1-\mathrm{cm}$ incision was made on her left temporal area, and a large amount of abscess was drained. Brownish yellow discharge with foul odor was spontaneously wiped off. In regard to the patient's medical conditions, the size of abscess, the degree of pain, the incision, surgical drainage under the general anesthesia was considered during the first treatment. The enhanced facial computed tomography (CT) showed a $48 \times 78 \times$

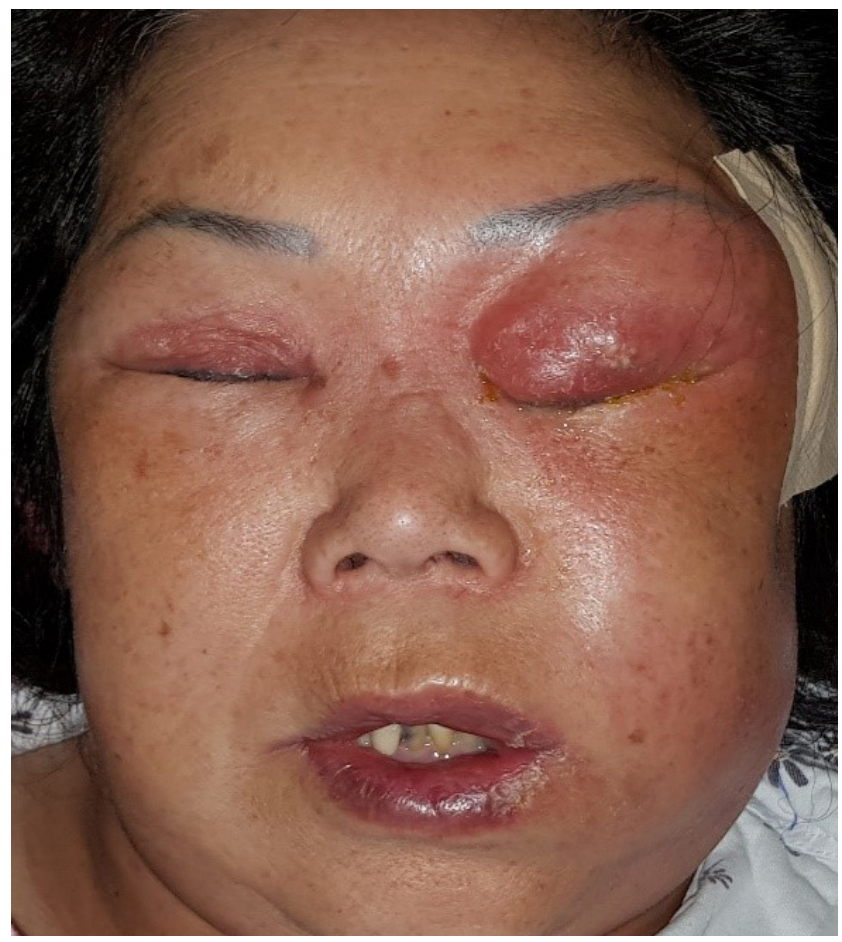

Fig. 1. Initial picture. At the time of admission, swelling, redness, and tenderness were observed.
$23 \mathrm{~mm}$ abscess mass on the patient's left, upper eyelid and left temporal fossa area (Fig. 2). At the time, the laboratory data showed the CRP level of $19.98 \mathrm{mg} / \mathrm{dL}$, and WBC at $9,600 / \mu \mathrm{L}$. The data revealed a 5 -fold increase in CRP from the previous data from the local clinic. Third-generation cephalosporin and aminoglycoside antibiotics were administrated intravenously.

The following day, the preoperative evaluation of the patient was reviewed. In the preoperative exams, solid nodule in the thyroid and deviated trachea, that the patient was unaware of, were found (Fig. 3). The anesthetics such as propofol and ketamine were administered with an intubation procedure. However, such a process was considered difficult and risky due to the trachea deviation of the patient. Consequently, local anesthesia was an alternative choice for the patient due to her medical conditions.

Since the local anesthetic was applied at the surgery, the patient was mentally alert with regular breathing. The patient inevitably suffered from significant pain, and her poor co-operation made it difficult to break the locules of the abscess. Incision, drainage with elongated incision and Irrigation were carried out to ensure a sufficient abscess removal. After the surgery, the patient's condition was closely monitored in cooperation with the anesthesiology department. The open incision

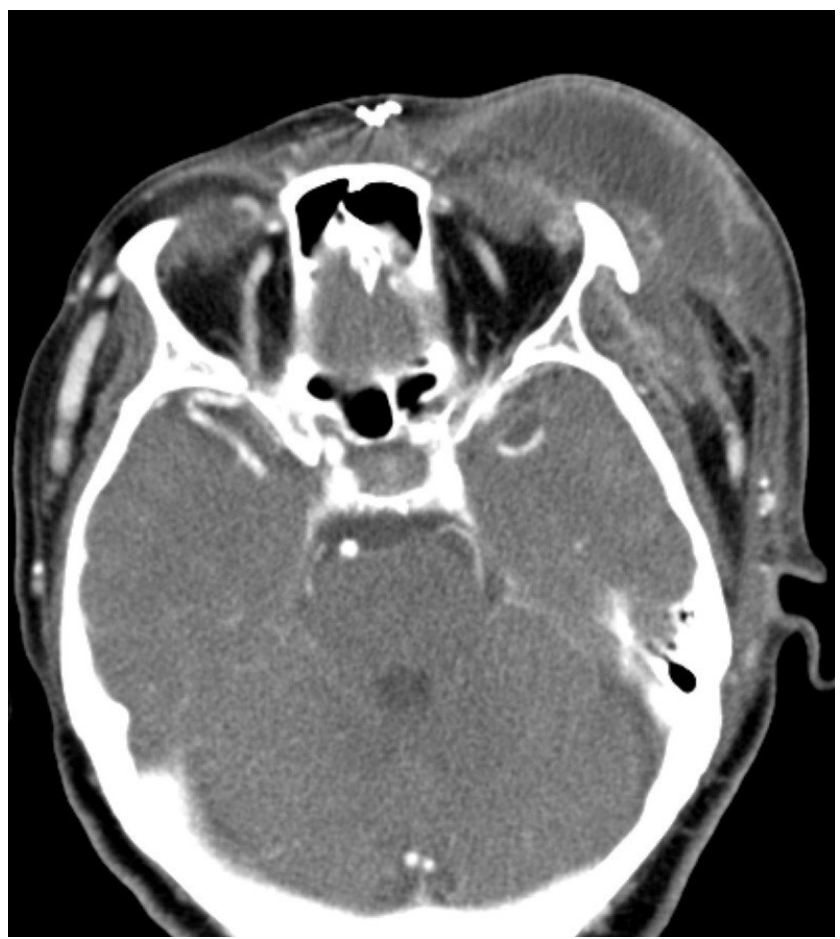

Fig. 2. Initial computed tomography (CT). Note to left periorbital abscess on enhanced facial CT at the time of admission. 

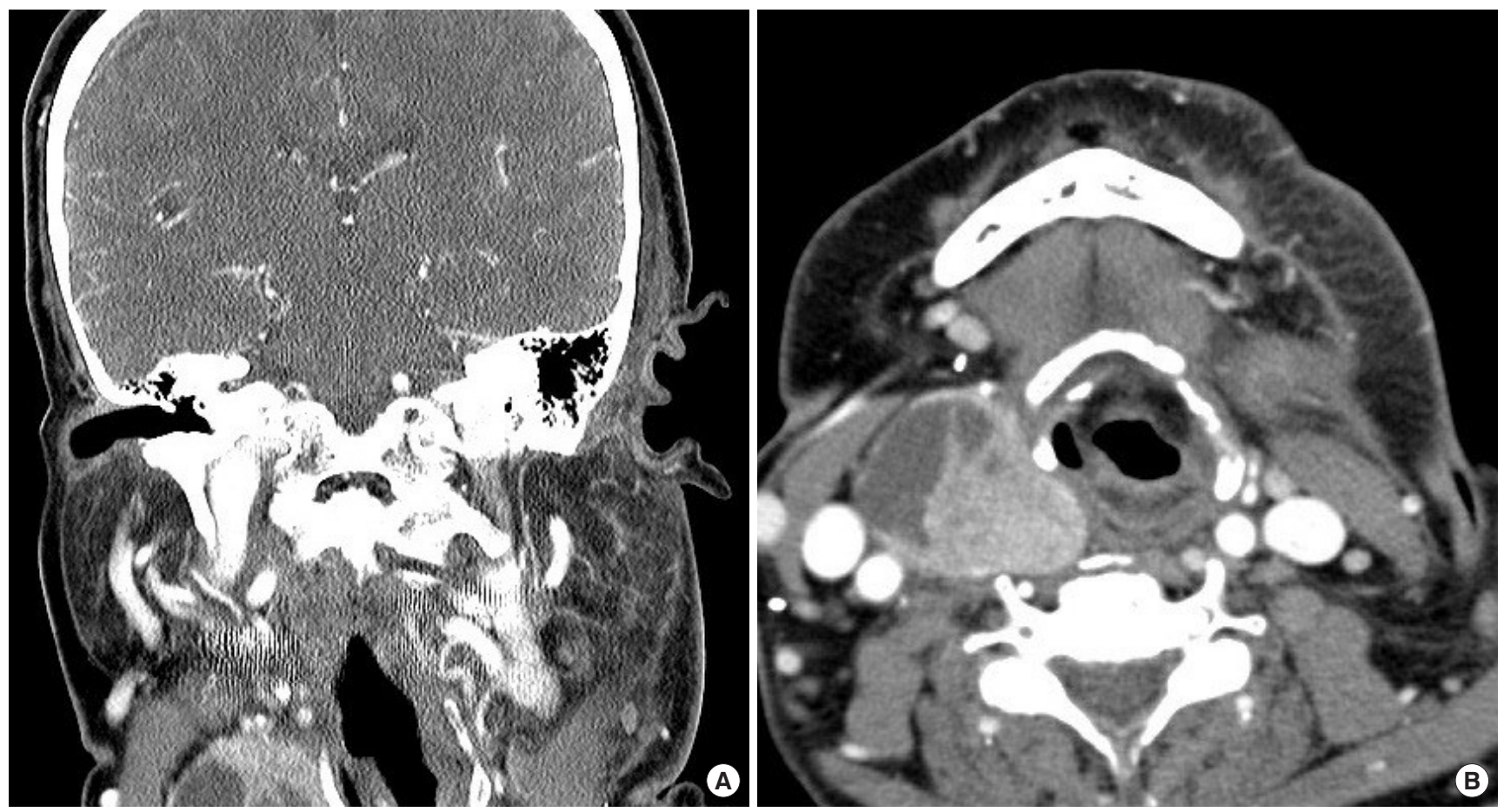

Fig. 3. Deviated trachea due to thyroid mass. (A) Coronal section. (B) Axial section.

site was dressed two or three times on a daily basis.

Due to a large amount of abscess on the left ocular region, a gauze packing with a betadine dressing was applied at the left eyelid area for sufficient drainage. The surgical drainage alleviated the inflammation symptoms, pain, and swelling. Furthermore, the postoperative pain control was started with simple nonsteroidal anti-inflammatory pain-control drug without fentanyl-based patient controlled analgesia.

The abscess was later identified as Streptococcus anginosus by bacterial identification. The initial antibiotics application was maintained [3,4]. CRP dropped to $2.84 \mathrm{mg} / \mathrm{dL}$ by day 7 after the surgery, and again to $0.45 \mathrm{mg} / \mathrm{dL}$ after day 14 . Facial edema was significantly decreased in size, and the patient's pain was relieved. On day 14 after the first incision and drainage, the debridement of inflammatory tissues on the incision area of the left cheek and eyelid was performed with sufficient irrigation, and then the wound of the left eyelid was sutured. On day 17 postoperation, the remaining inflammatory tissues in the left cheek were removed, and the wound area was sutured. The medication was changed to the first-generation, cephalosporin-based per oral. The stitches were taken out 5 days' post-suture, and the patient was discharged with a full recovery. A follow-up check-up was conducted on days 2 and 5 of post-discharge, and no symptoms of additional edema or

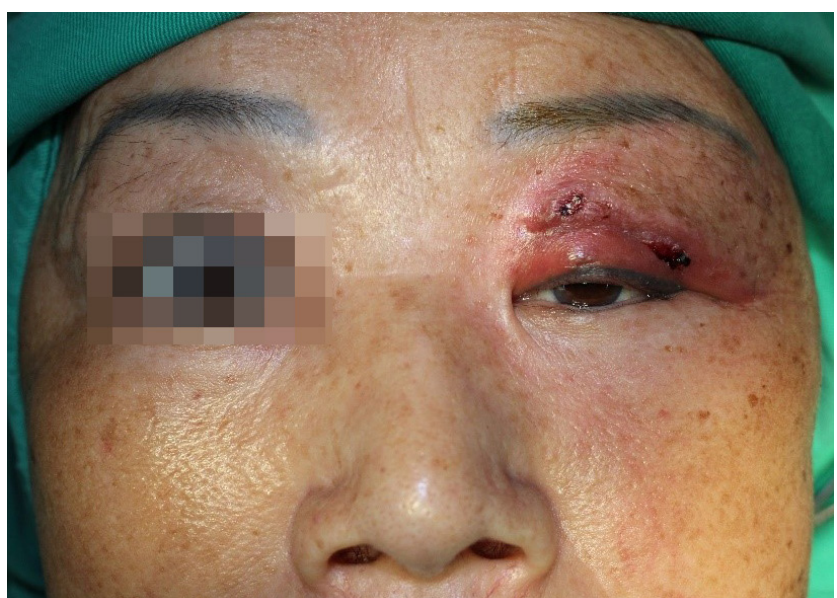

Fig. 4. Blepharoptosis aspect appearing on the left-eye side.

inflammatory response were observed.

After 8 months since the discharge, the patient has been satisfied with all of the treatments since the patient has no symptoms of pain, swelling, ptosis, and facial asymmetry.

\section{Discussion}

Drainage, curettage, and packing have sequentially proceeded in conventional superficial abscess treatments [5]. However, in 
this case, the patient's medical condition limited the use of general anesthesia, making it difficult to extract the entire abscess in a single operation. Local anesthesia was applied in the stepwise abscess removal surgery. The multiple dressing changes and antibiotic treatment were necessary for the more effective and complete recovery of the patient [6]. The abscess $(48 \times 78 \times 23 \mathrm{~mm})$ was formed around the left periocular and temporal area, and the CRP and WBC were increased to a high level at the time of admission. After the incision and drainage treatments, the patient was treated with continuous dressing to prevent further deterioration; for example, "sepsis." The abscess on the left upper eyelid was drained clearly before it was developed into "conglutination" which might lead to the blepharoptosis at the time of discharge (Fig. 4).

General anesthesia was initially considered due to the size of the abscess and the severity of the symptoms. However, a huge thyroid mass was found in the enhanced facial CT, which was originally conducted to precisely measure the abscess. The large mass on the thyroid caused trachea deviation via compression. The use of tracheal intubation and general anesthetics was critically questioned and not favored by many staffs. Indeed, local anesthesia was used on the patient for safety concerns.

The patient's obesity, long duration of the disease, and the severe symptoms of inflammation further led to believe that the risk of general anesthesia was too high [7]. A huge mass over $6 \mathrm{~cm}$ diameter may cause tracheal deviation. Moreover, it is more likely to block the narrowed airway when a neuromuscular blocker is used. Since a further evaluation of the thyroid mass was required, the possibility of underlying thyrotoxicosis should be considered. Therefore, the following stepwise surgical approach was recommended: incision and drainage with local anesthesia [8]. In the operation, lidocaine was used for local anesthesia. Overall, the patient was well handled with effective treatment.

In many regions, acupuncture is believed to have significant effects in reducing pain and swelling $[9,10]$. Nevertheless, the cases of side effects, such as an infection, have also been reported [11-13]. In some cases, sepsis is caused by infection [14]. The patient admitted to our team is a case in which a healthy woman has suffered from a severe abscess formation that was caused by a single acupuncture treatment. The abnormality of the patient's WBC level along with her blood was severe enough to diagnose SIRS.

In conclusion, as a medical doctor looking into a patient suffering from an abscess or an infection should find out if there have been any histories of acupuncture treatments. It is most important for the patients to have surgical drainage as soon as possible.

\section{Conflict of interest}

No potential conflicts of interest relevant to this article are reported.

\section{Acknowledgments}

Kwan Sub Kim https://orcid.org/0000-0001-7868-1713

Wu Seop Lee https://orcid.org/0000-0002-7540-4259

Sung Min Shim https://orcid.org/0000-0001-7530-367X

Yong Min Kim https://orcid.org/0000-0001-6866-4135

So Young Ji

https://orcid.org/0000-0002-6060-1452

\section{References}

1. Taub D, Yampolsky A, Diecidue R, et al. Controversies in the management of oral and maxillofacial infections. Oral Maxillofac Surg Clin North Am 2017;29:465-73.

2. Salehi A, Marzban M, Imanieh MH. The evaluation of curative effect of acupuncture: a review of systematic and metaanalysis studies. J Evid Based Complementary Altern Med 2016;21:202-14.

3. Han B, Choi MW, Han TY, et al. A case of mycobacterium chelonae infection at the site of acupuncture. Korean J Dermatol 2017;55:717-20.

4. Kim JH, Seunghwan OH, Jun JY, et al. Cutaneous Mycobacterium massiliense infection associated with acupuncture. Korean J Dermatol 2016;54:75-6.

5. Abraham N, Doudle M, Carson P. Open versus closed surgical treatment of abscesses: a controlled clinical trial. Aust N Z J Surg 1997;67:173-6.

6. Schramm VL Jr, Curtin HD, Kennerdell JS. Evaluation of orbital cellulitis and results of treatment. Laryngoscope 1982; 92(7 Pt 1):732-8.

7. Martinez-Casas I, Sancho JJ, Nve E, et al. Preoperative risk factors for mortality after relaparotomy: analysis of 254 patients. Langenbecks Arch Surg 2010;395:527-34.

8. Bouaggad A, Nejmi SE, Bouderka MA, et al. Prediction of difficult tracheal intubation in thyroid surgery. Anesth Analg 2004;99:603-6.

9. Felix ER. Chronic neuropathic pain in SCl: evaluation and treatment. Phys Med Rehabil Clin N Am 2014;25:545-71.

10. Borg-Stein J, laccarino MA. Myofascial pain syndrome treat- 
Kim KS et al.

Post Asian acupuncture infection case

ments. Phys Med Rehabil Clin N Am 2014;25:357-74.

11. Zhang J, Shang H, Gao X, et al. Acupuncture-related adverse events: a systematic review of the Chinese literature. Bull World Health Organ 2010;88:915C-921C.

12. Cho HJ, Lee DY, Lee JH, et al. A case of Mycobacterium abscessus skin infection caused by multiple acupunctures. Clin Exp Dermatol 2010;35:444-5.
13. Nakajima A, Kaneyama $\mathrm{R}$, Watanabe $\mathrm{H}$, et al. Acupuncture needle-associated prosthetic knee infection after total knee arthroplasty. Mod Rheumatol 2010;20:627-31.

14. Maas ML, Wever PC, Plat AW, et al. An uncommon cause of Staphylococcus aureus sepsis. Scand J Infect Dis 2013;45: 722-4. 